\title{
Index to Volume 8
}

Numbers followed by asterisk refer to abstracts

Abaci, F. $461 *$

Abate, M. 422*

Abita, J.-P. 714

Abramson, M. B. 366*

Aceto, T., Jr. 373,*375*

Acid hydrolases 774

Ackerman, B. D. 443*

Ackerman, I. 452*

Acrodermatitis enteropathica 82

Adam, P. 370*

Adcock, E. W. 360*

Addo, N. $367^{*}$

Adebonojo, F. O. 356,*905*

Adelman, R. D. 378*

Adelson, J. W. 381 *

Adenine arabinoside 825

Adenine phosphoribosyltransferase 31

Adenosine arabinoside 945

Adenosine 3',5'-monophosphate 37, 223

$S$-Adenosylmethionine decarboxylase 231

Adenylate cyclase 244

Adipose tissue 800

Agustin, A. V. $367^{*}$

Alanine 951

Albumin 963

Alford, C. A. $423, * 428, * 430 *$

Alkjaersig, N. 456,* 460*

Allen, A. C. $443^{*}$

Allen, D. O. 223

Allen, H. D. $342, * 343, * 349, * 352 *$

Allen, J. 399*

Allen, R. J. 396*

Alling, D. W. 422*

Alpert, E. 392*

Alpert, S. 428*

Alt, C. $362 *$

Altman, A. J. $397^{*}$

Alvarado, J. 385*

Alvarez, M. N. 453*

Amato, D. $397^{*}$

Amino acids 598, 697, 830, 884

Amino acid transport 673

$\alpha$-Aminoisobutyric acid 697

Ammann, A. J. 413, 420 *

Amniocentesis 746,870

Amniotic fluid 553, 591, 679

Amniotic fluid cells 870

Ampaya, E. P. $412 *$

Ampola, M. G. 387 *

Anaerobiosis 884

Anand, S. K. 368*

Andersen, R. N. 369*

Anderson, G. H. 379*

Anderson, P. A. W. 347*

Andersson, R. 852

Andrews, B. F. 378,* $443 *$

Andrews, L.-L. 428*

Androgen 103

Andronian, S. 402*
Anemia 176

Angiography 561

Angiotensin 611

Angle, C. R. 397*

Anoxia 238

Antidiuretic hormone 792

Antinuclear antibodies 806

Antipyrine 796

Antonowicz, 1. 383*

Anttila, R. O. 901*

Aperia, A. 758

Apoceruloplasmin 821

Applebaum, M. N. 401*

Arachidonic acid 82, 93

Aranda, J. V. 362*

Arant, B. S., Jr. 638

Arbeter, A. M. 689

Arbogast, B. 436*

Arcilla, R. A. 67, 348*

Arginine esterase 684

Arias, I. M. 591

Arnold, A. 18

Aronson, A. S. 895*

Arylsulfatase A 865

Asch, H. 346*

Ashwal, S. 945

Aschinberg, L. C. 453*

Asnes, R. S. 422*

Asphyxia 50, 58

Aspnes, G. T. 405,* 406*

Assisted ventilation 621

Asthma 689

Atherton, H. 451*

Athreya, B. H. 397 *

ATP 238

ATPase 594

Atwater, J. 955

Aubert, M. L. 375*

Auerbach, V. H. 455, $* 561$

August, C. 412 *

Auld, P. A. M. $452, * 464$ *

Aur, R. 409*

Austin, S. R. 424*

Avery, M. E. 467,* 471*

Avery, S. 223

AvRuskin, T. W. 367*

Ayoub, E. M. 418*

Azarnoff, D. L. 364*

Azotemia 163

Azzi, R. 359*

Bachmann, C. $906, * 907$ *

Bacteremia 718

Badger, E. 342*

Baehner, R. L. 411 *

Baez, S. 403*

Bagnell, P. $430^{*}$

Bahoric, A. $465^{*}$

Bakay, B. 31
Balachandar, V. 371,*377*

Baldwin, L. S. $377^{*}$

Balistreri, W. F. 378*

Ballabriga, A. 89,93

Ballard, C. 422*

Ballard, P. L. $358, * 367, * 382 *$

Ballowitz, L. $912 *$

Balsan, S. $897^{*}$

Baluarte, H. J. 455*

Bancalari, E. 347,* 448, * 468*

Barajas, L. 611

Bard, H. 385,*397,* 400,* 443*

Barlow, B. $384 *$

Barltrop, D. 915*

Barnes, N. D. 453*

Barness, L. 436*

Barness, L. A. 436*

Barnett, D. R. 687

Barqman, G. J. 464 *

Barr, M., Jr. 447*

Barrat, T. M. $901 *$

Barrett, C. T. 244, 362*

Barron, R. $407^{*}$

Barth, A. 380*

Bartholome, K. 906*

Barton, L. 422*

Bartsocas, C. S. 673

Bashore, R. A. 611

Bass, F. 442*

Bass, J. W. 412,* 421,* 430*

Bassett, A. L. $350^{*}$

Batchelor, J. R. 412*

Battaglia, F. C. $360, * 830$

Battles, M. 442*

Battles, N. D. $460^{*}$

Baud, R. 897*

Bauer, C. R. 405*

Bauer, R. 611

Baumgartner, R. 906,*907*

Bautista, J. 379*

Baxter, J. D. $367^{*}$

Baylen, B. $347^{*}$

Beach, R. L. 469*

Bean, J. A. 403*

Beaudry, M. A. $360, * 374 *$

Beaudry, P. 466*

Beaudry, P. H. 468 , * 470 *

Bebin, J. 462*

Becker, K. 82

Bednarek, F. J. 444 *

Begemann, P. H. 114

Behrman, R. E. 449*, 792

Beisel, W. R. 13

Belin, R. P. $369^{*}$

Bell, G. F. 382*

Bell, N. H. 223

Bellanti, J. A. $413, * 420 *$

Bendien, C. $910^{*}$

Benenson, A. S. 422 * 
Benjamins, D. 462*

Bennett, B. $454, * 459 *$

Bennett, S. 366*

Beno, P. 914,* 917 *

Benz, E. J., Jr. 387*

Beran, A. V. $443^{*}$

Beratis, N. G. $388, * 390,{ }^{*} 687$

Berend, K. 916*

Berger, M. 378,* 454*

Bergstrom, W. H. $437, * 439, * 448 *$

Berk, P. D. 573

Berle, B. B. $364^{*}$

Berman, J. L. 389*

Berman, P. 346*

Berman, W. 379*

Bernard, B. 422 *

Bernfield, M. R. 184, 356*

Bernstein, J. 453,* 459*

Bernstein, J. M. $415^{*}$

Bernstein, R. A. 467*

Bersu, E. T. 439*

Bessinger, F. B., Jr. 347*

Bétend, B. 443*

Beutner, K. 415*

Bhakdi, S. $910^{*}$

Bhatnagar, A. S. 378*

Bhupathy, K. 443*

Bicarbonate 735

Bieler, E. 918*

Bienzle, U. 900*

Biggar, W. D. 397*

Bile 710

Bilirubin 573, 963

Bilirubin encephalopathy 647

Billmeier, G. J., Jr. 809

Binkiewicz, A. 438*

Bjornson, L. 796

Black, I. F. S. $455^{*}, 561$

Black, M. A. $343^{*}$

Blanc, W. A. 200, 356*

Blanchard, M.-G. 968

Blanco, C. E. 444*

Bland, R. D. 464*

Blandin-Savoja, 540

Blaschke, T. F. 573

Blatteis, C. M. 809

Blattner, P. 397*

Blau, E. B. 453*

Blaufox, M. D. 455*

Bloch, K. J. 420*

Blood flow, 257, 561

Bloom, A. D. 387 *

Blum, A. $909 *$

Blume, K. G. 18

Blumenfeld, T. A. $356^{*}$

Blumenthal, S. $350^{*}$

Boat, T. F. $422, * 465, * 531,861$

Bobik, C. 435*

Bobik, J. 435*

Bocala, R. R. 347 *

Boches, F. 440*

Boda, D. 900*

Bode, H. H. 445*

Boehm, J. 789

Boerth, R. C. $362 *$

Boime, I. 770

Boineau, F. G. 453*

Boley, S. J. 380*

Bommer, J. 913*
Bonforte, R. J. 687

Bongiovanni, A. M. $370,{ }^{*} 371, * 372, *$ $376,{ }^{*} 377, * 407 *$

Bonnet, F. 800

Book, L. S. 379*

Booth, C. W. 387*

Borden, M. 31

Borer, R. C., Jr. 444,* 447 *

Borkon, A. M. 347*

Bortolussi, R. 379*

Bosshard, H. R. 704

Bowe, E. 270

Bowe, E. T. $450,{ }^{*} 454$ *

Bowie, M. D. 879

Bowman, B. H. 687

Boxerbaum, B. 422 *

Boyar, R. M. 378*

Boyd, R. 899*

Boyd, T. H., III 75

Boyer, K. M. 422*

Bradley, R. M. 391*

Brady, R. O. $391 *$

Brain $89,93,120,238,263,714,718,945$

Brain development 783

Branca, P. A. $445,{ }^{*} 465^{*}$

Brandis, C. M. $894^{*}$

Brandt, C. D. $415, * 422 *$

Brandt, E. J. $371^{*}$

Brandt, I. K. 388*

Brann, A. W., Jr. 462*

Brans, Y. 447*

Brans, Y. W. 215

Brauser, B. $907^{*}$

Bray, G. A. 368 , $432 *$

Brazelton, T. B. $342^{*}$

Bremer, H. J. 904*

Breslow, J. L. 387*

Bresnan, M. 435,* 438*

Bretscher, D. $899^{*}$

Brickman, A. 345*

Brinsfield, D. E. $355^{*}$

Brinson, A. O. $448^{*}$

Brinson, P. $390^{*}$

Broberger, O. 758

Brodehl, J. 893,* 894*

Brook, C. G. D. 248

Brown, D. 451*

Brown, D. M. $416^{*}$

Brown, E. G. $383, * 444,{ }^{*} 448 *$

Brown, J. A. 371*

Brown, W. R. 815

Broyer, M. 901,*903,*913*

Brumley, G. W. 429*

Brun Del Re, S. $909^{*}$

-Brunell, P. A. 945

Bryan, A. C. $465, * 466^{*}$

Bryan, E. M. $915^{*}$

Bryan, M. H. 379 ,* $465 *$

Bryant, E. M. 746

Bryant, K. 394*

Brych, M. 373*

Bryson, M. F. 459*

Buchanan, R. A. 423*

Budzilovich, G. 945

Buimovici-Klein, E. 420*

Bullock, W. H. 402*

Burdine, J. 355*

Bureau, M. 470*

Burgert, E. O., Jr. 360,* $398 *$
Burke, B. A. 452 *

Burke, J. A. $454^{*}$

Burke, J. P. 424 *

Burmeister, L. 460*

Burns 931

Burns, D. C. 342 *

Burton, B. K. 170

Butcher, R. 463*

Byfield, P. E. 419*

Byrd, D. J. $906 *$

Byrne, P. A. $465^{*}$

Cabal, L. 444, * 452*

Cahill, L. T. $411, * 414 *$

Calcagno, P. L. 456, ${ }^{*} 458 *$

Calcium 531

Caldroney, G. 423*

Callahan, J. 393*

Callahan, J. W. 387*

Callis, L. 895*, 913*

Camisa, C. $398^{*}$

Camitta, B. $471^{*}$

Canales, L. 420*

Cancer chemotherapy 75

Cancilla, P. 393*

Cann, H. M. 5, 388*

Cantez, T. 354*

Cantwell, R. J. $342 *$

Cantz, M. 904,* $916 *$

Capino, A. G. $449 *$

Capodanno, C. C. $365^{*}$

Carbohydrate metabolism 13

Carlile, W. K. $445 *$

Carlos, E. $398, * 437 *$

Carpenter, G. 462*

Carrel, R. E. 368*

Carson, J. 905,* $908 *$

Carson, N. A. J. 42

Carvajal, H. F. 454*

Casagrande, S. $425^{*}$

Cassady, G. 215, 447*

Cassady, R. $350 *$

Cassiman, J.-J. 184, 356*

Castello, F. 895,* 913 *

Castells, S. 367, * 461*

Castro, S. 448*

Catch-up growth 149, 157, 929

Cates, D. B. 450 *

Cates, M. 388*

Cathelineau, L. 857

Cation transport 594

Caviness, V. S., Jr. 546

Cejka, J. 403,* 414*

Cell adhesion 184

Cell aggregation 184

Cell fusion 606

Cerebellum 843

Cerebral blood flow 50

Cerebrum 50, 58

Cevallos, E. 459*

Chacko, C. M. $388^{*}$

Chamales, M. H. 401*

Champlin, R. 427*

Chan, D. 402*

Chan, G. 398*

Chan, J. C. M. $383, * 454, * 457 *$

Chance, G. W. $360, * 438, * 452 *$

Chang, D. C. $359 *$

Chang, H. 398*

Chanock, R. M. 415,* 977 
Chantler, C. 109

Chapa, I. A. $420 *$

Chaplin, E. R. $462 *$

Chappuis, M. C. $898^{*}$

Charpentier, C. 857

Chartrand, L. 385*

Chase, H. P. $379, * 386, * 431, * 432, * 462 *$

Chase, P. A. 417 *

Chavez, C. J. $365, * 449 *$

Chen, I.-W. 376*

Chen, J. C. $444^{*}$

Chen, J. H. $348,{ }^{*} 445, * 465 *$

Chen, S.-H. $395 *$

Chen, S. Y. $371, * 377 *$

Chen, Y. 469*

Chernack, W. J. 422*

Chernick, V. $465^{*}$

Cherry, J. D. 422 * $463 *$

Chesney, R. W. $348, * 431, * 454 *$

Cheung, W. M. 377*

Ch'ien, L. T. $423 *$

Chilcote, R. R. 398,* 417 *

Chiroff, R. T. $439 *$

Chiswick, M. L. 908*

Chloralose 58

Chloride space 270

Choinière, L. 399*

Cholesterol 170, 704

Choline 848

Choline phosphoglycerides 93

Chou, L. 120

Christiansen, R. O. $431^{*}$

Christine, B. 342*

Chung, L. $366^{*}$

Churg, J. 454*

Chytil, F. 470*

Cineangiocardiography 67

Citrulline 42

Cividalli, G. 553

Clark, C. M., Jr. 223

Clarke, S. 402*

Clemens, L. E. 370 *

Clements, J. A. $358^{*}$

Clemons, R. D. $367^{*}$

Clendenin, J. 402*

Clyde, W. A., Jr. $424 *$

Coen, R. W. 444*

Cohen, A. H. $368^{*}$

Cohen, M. I. $380, * 392, * 405 *$

Cohn, H. E. 348*

Cole, B. R. 456,* 460*

Cole, H. S. $379^{*}$

Collier, A. M. $424, * 425 *$

Collins, D. $469^{*}$

Collipp, P. J. $371, * 377, * 465 *$

Collu, R. 368*

Colon, A. R. 462*

Colostrum 815

Colten, H. R. 421 *

Conde, C. 89,93

Condie, R. M. 421*

Congenital disease 945

Congenital heart disease 561

Congenital metabolic disease 18, 26

Congenital nonhemoly tic jaundice 573

Congenital nonspherocy tic anemia 26

Congenital nonsperocytic hemolytic anemia 18

Connaughton, J. F. 363*

Connor, J. D. $422, * 825$
Conod, E. J. 687

Conover, J. H. 687

Conte, F. A. 373*

Cook, C. D. $472 *$

Cooper, D. $466^{*}$

Cooper, D. M. $466^{*}$

Cooper, J. 921

Cooper, L. Z. $420^{*}$

Cooper, M. D. 421*

Cooperman, J. M. 379*

Copper deficiency 821

Coproporphyrin 591

Corash, L. 398,* 770, 774

Corbeel, L. M. 721

Corbet, A. $466^{*}$

Corbin, A. 435*

Corby, D. G. 399*

Cordero, L. 444*

Cornblath, M. 391,*466,* 469*

Cornfeld, D. 370*

Corrigan, J. J., Jr. 399,* 402*

Cosgrove, J. 466*

Costiloe, P. 383*

Costin, G. 367, * $368^{*}$

Cote, M. L. $455^{*}$

Cotton, M. 393*

Coulombe, J. T. 434*

Counts, G. 429*

Courpotin, C. $445^{*}$

Covell, J. W. 351*

Cowett, R. M. $445^{*}$

Cowger, M. L. 431*

Cowles, C. $435^{*}$

Cox, B. J. 75

Cox, D. M. 679

Cox, D. W. 466*

Cox, R. P. 606

Coyer, W. F. 423*

Cracco, J. B. $462 *$

Cracco, R. Q. 462*

Craig, L. 392,* 393*

Crane, L. A. 470*

Crawford, J. A. 416*

Crawford, J. D. 359,* 368,* 375,* 673

Crigler, J. F., Jr. 431 *

Crigler-Najjar syndrome 573

Crocker, J. F. S. 423*

Crosby, W. 383*

Crozier, D. N. $349 *$

Crying 921

Cser, A. 911*

Cukier, J. O. 399*

Cullen, P. 386*

Cunningham, K. 349,*354*

Curran, J. 436*

Curran, P. F. $380, * 898, * 909 *$

Curran, W. J. $471 *$

Curtius, H. C. 704

Cutilletta, A. F. 348*

Cvoric, A. 895*

Cystic fibrosis of the pancreas 205, 212, $531,594,684,861$

Cytosine arabinoside 825,945

Czapek, E. E. 355*

Daily, W. J. R. 445*

Dajani, A. S. 423 *

Dallaire, L. 388 * $392 *$

Dallman, P. R. $409^{*}$

Dancis, J. 606, 796
Danesh, B. J. N. Z. 894 *

Danesino, C. $390^{*}$

D'Angio, G. J. 408*

Daniel, S. S. 454*

Danielson, G. K. 347*

Danilowicz, D. 352*

Danner, D. J. 431*

Danon, M. $368, * 445 *$

Dansylcadaverine 789

Dar, H. 395, * 397*

Darby, W. E. 385*

Dartois, A. M. $897 *$

David, L. 898*

David, R. B. 436*

David-Faridy, M. F. 411*

Davidow, B. 345*

Davis, H. T. 870

Davis, S. D. $416^{*}$

Davis, W. J. 362*

Day, N. K. 901 *

Dechaux, M. 903*

Deforest, A. $413, * 423 *$

de Groppa, M. I. 373*

De Jong, M. C. J. $896^{*}$

DeLamater, P. V. $356, * 374, * 439 *$

deLannoy, C. W. $458, * 459 *$

Delaporte, C. $913^{*}$

de la Torre Verduzco, R. $450 *$

deLemos, R. A. 380, * 466*

Delivoria-Papadopoulos, M. $64,348,{ }^{*} 356$, $363, * 445, * 465 *$

Dell, R. B. 270, 609

DeLuca, H. F. 454*

DeLuca, K. J. 431*

Delvin, E. E. $381, * 893 *$

Demant, F. 914*

Demling, R. H. 464*

DeMonterice, D. $343 *$

de Morais, J. S. 429*

Denniston, J. C. 13

Depper, R. 697

Der Kaloustian, V. M. 9.09*

de Rosas, F. 106

Desjeux, J. F. 380,* 898 ,* $909 *$

Development 9.21

Development biochemistry $31,37,64,193$, $231,244,765,770,874$

Developmental physiology 270, 638, 758, 792

Devereux, D. F. $375^{*}$

DeVivo, D. C. $463^{*}$

Devlin, J. $356^{*}$

DeVries, G. H. 436*

Deyhle, P. $909^{*}$

D'huyvetter, P. 444*

Diamond, I. $462 *$

Diamond, M. 411*

Diaz, R. 408*

Diaz-Bensussen, S. 735

Dietzman, D.E. 423*

Di Ferrante, N. 388*

DiGeorge, A. M. 373*

Digout, S. C. 423*

Dilinoleyl lecithin 1

Dillon, M. J. 903*

Dimich, I. 445*

DiPaolo, R. V. 546

2,3-Diphosphoglycerate 64

Diven, W. F. 393*

Djatchcova, A. 914* 
DNA 806

Dober, I. 911*

Dobos, M. 915*

Dobson, V. 445*

Dodson, W. E. 362*

Doehmann, U. 905*

Doershuk, C. F. 422,* $465 *$

Doherty, R. A. 870

Dolovich, J. 469*

Dolovich, M. B. 469*

Donaldson, M. H. 407*

Donath, A. $897 *$

Donnelly, P. V. 388*

Donnelly, W. H. 455 * 460 *

Dooley, R. 435*

Doray, B. 385*

Doray, B. H. 399 , * 443*

Dorche, C. 714

Dorfman, A. 436*

Dowd, E. 451*

Dowell, R. T. 348*

Dowling, S. 343*

Downing, T. 402*

Doyle, E. F. 352*

Drachman, R. H. 412*

Drash, A. $435, * 467 *$

Dreborg, S. 852

Drexler, E. 806

Driscoll, S. 428*

Drukker, A. 454,* 455*

Drummond, K. N. $348^{*}$

Drummond, W. H. 348*

Dube, S. K. 348*

Dublin, P. A., Jr. 388*

DuBois, A. B. $445, * 465 *$

Dubowitz, V. $907 *$

Ducharme, J. R. 368*

Duckett, G. 375*

Duckett, G. E. $374, * 455 *$

Duffield, A. 446*

Duffy, T. E. 464*

Dukes, P. P. 398*

Dunn, L. J. $378^{*}$

Dupont, C. $399 *$

Dupree, E. $412 *$

Durie, B. G. 418 * $419 *$

Dweck, H. S. 215

Eade, N. R. $362 *$

Eck, E. 900*

Edelmann, C. M., Jr. $453, * 455, * 638$

Eeckels, R. 721, 800

Eeg Olofsson, O. 911*

Egan, E. A. $466 * 467 *$

Eggermont, E. 721

Eggleston, A. W. $412 *$

Eggleston, P. A. $412, * 466 *$

Egli, F. 903*

Eicher, E. M. 389,* 899*

Eidelman, A. 424*

Eife, G. $412^{*}$

Eife, R. 412*

Eisenstein, T. D. 410 *

Eisner, G. M. 456*

Eitzman, D. V. 467*

Ejection fraction 67

Elders, M. J. 371 *

Elema, J. D. 910 *

Ellenberg, J. H. $418^{*}$

Ellis, E. F. 689
Elmiger, P. 903*

Elsas, L. J. 431*

Embil, J. A. 628

Embryonic cells 184

Emich, J. P. 363*

Emilio, M. G. 917*

Emmanouilides, G. C. 354*

Emotional trauma 931

Enders, J. F. 424*

Endotoxin 13

Endreffy, E. 900*

Engbring, J. 443*

Engel, R. R. 381,* 467*

Enger, E. 899*

Epps, C. H., Jr. 400*

Epstein, M. F. 356,* 446*

Epstein, M. L. 349*

Epstein, R. A. 468*

Epstein, S. 363*

Epstein, S. E. 352*

Erenberg, A. 783, 951

Ernest, M. A. 439*

Erythrocytes(s) 64, 176, 594

Erythropoiesis 176

Erythropoietin 176

Escherichia coli 815,935

Eshaghpour, E. 353*

Eshai, R. 433*

Esterly, J. R. 380* $384 *$

Estrogen 740

Ethanolamine phosphoglycerides 93

Evans, B. C. $353 *$

Everett, J. R. 424*

Evoked potentials 58

Exeni, R. A. 380*

Eyring, E. J. 404*

Faden, H. S. 424*

Fagenholz, S. A. 470*

Fairbanks, V.F. $360, * 398 *$

Fallat, R. 434*

Falletta, J. M. 400*

Fallstrom, K. 911*

Familial hypercholesterolemia 170

Fanaroff, A. 463,* 468*

Fang, V. S. $374 *$

Farrell, M. A. 468*

Farrell, P. M. 205, 356,* 446,* 452,* 467*

Farriaux, J. P. 393*

Farzaneh, I. 422*

Fat 215

Fathalla, M. 380*

Fatty acid synthesis 193

Faulkner, S. L. 362*

Feck, J. C. $390^{*}$

Federlin, K. 897*

Feingold, M. 441*

Fekete, G. 915*

Feldman, R. A. 429*

Feldman, S. 426*

Feldt, R. H. 347*

Feig, S. A. 594

Felix, J. S. 870

Fellows, F. C. I. 42

Felt, R. L. 453*

Fennell, R. S. $455, * 460 *$

Fennell, R. S., III 424, $455 *$

Fenoglio, J. J. 350*

Ferdinandus, L. D. 884
Fernald, G. W. $424, * 425 *$

Fernandes, J. 114

Fernbach, D. J. 400*

Fernbach, S. 446*

Ferrer, P. L. $349, * 350 *$

Ferrieri, P. 424*

Ferry, G. 382*

Fetal calf serum 870

Fetal hemoglobin 64

Fetus $37,58,200,231,244,553,611,697$, $714,755,765,783,789,796,830,848$, $951,960,968$

Fibrinolysis 75

Fibrin-stabilizing factor 75

Fibrin-stabilizing factor (factor XIIn) 789

Fikrig, S. M. 394*

Filer, L. J., Jr. $386^{*}$

Finberg, L. 460*

Fine, B. P. $380^{*}$

Fine, R. N. $343, * 412, * 458 *$

Finegold, M. 945

Finegold, R. M. 430*

Fink, C. W. $460^{*}$

Finkelstein, J. W. $378, * 382 *$

Finklestein, J. Z. $400 *$

Finnegan, L. P. $344, * 346, * 363, * 364$ *

Fireman, P. 467*

Fischer, G. W. $412 *$

Fiser, R. H. 13

Fiser, R. H., Jr. $356, * 368, * 432, * 434, *$ $439, * 951$

Fish, I. 945

Fishaut, J. M. 825

Fisher, D. A. $356, * 368, * 374, * 432, *$ $439, * 610,783,951$

Fisher, D. E. $386, * 449, * 459, * 792$

Fisher, L. 390*

Fisichelli, R. M. 921

Fisichelli, V. R. 921

Fitzgerald, J. F. 884

Fitzhardinge, P. M. 446*

Fixler, D. E. $349^{*}$

Fleischman, A. R. 446*

Fleisher, D. S. $343^{*}$

Fleisher, L. D. $388^{*}$

Fletcher, A. P. $456, * 460$ *

Flores, N. 385*

Florio, L. 419*

Fluoxymesterone 103

Foley, T. P., Jr. 367*

Forbes, G. A. 929

Forbes, G. B. $344, * 459 *$

Ford, R. C. $389 *$

Forget, B. G. 387 *

Forget, P. P. 114

Forster, R. E. $356,{ }^{*} 445 *$

Forster, R. E., II 64, 348*

Fosbrooke, A. 908*

Fowler, R. 354*

Fowler, R. S. 349*

Fox, G. N. $467^{*}$

Fox, H. A. $446, * 450$ *

Fox, W. W. 470*

Frable, W. J. 378*

Fraher, T. 418*

France, T. D. 440 *

Francois, R. 898*

Franks, R. 368*

Frantz, I. D., III $381, * 467$ *

Franz, H. E. 897* 
Fraser, F. C. $396, * 441 *$

Frasier, S. D. $372, * 407 *$

Freed, M. D. 349*

Freedman, M. H. 397*

Freedom, R. M. 348, *349,*352*

Free fatty acids 796

Freeman, R. B. 459*

Freeman, R. K. 200

Frei, E., III 402*

French, M. E. 412*

Frenkel, L. D. 413*

Frézal, J. 540

Frias, J. $418^{*}$

Frias, J. L. $441, * 442 *$

Friday, G. A. $467 *$

Friedman, G. 342 ,* $343 *$

Friedman, G. D. 346*

Friedman, G. M. $342 *$

Friedman, M. 417*

Friedman, M. A. 376*

Friedman, S. $408, * 955$

Friedman, W. F. 351,*354*

Freidman, T. 358*

Frigoletto, F. 553

Frigoletto, F. D. 391, * $392 *$

Froberg, L. 368*

Fructokinase 765

Fry, L. K. 386*

Fu, Y.-S. 405*

Fuccillo, D. A. $420, * 430 *$

Fukanaga, K. 5

Fulginiti, V. A. $418, * 419$ *

Fulwiler, R. 931

Funderburk, S. 393*

Gabbay, K. H. 431*

Gacs, G. $916^{*}$

Gaines, J. 342,*343*

Gajl-Peczalska, K. J. 403*

$\beta$-Galactosidase 120

Galasso, G. J. 422*

Galet, S. 400*

Gall, E. P. $459^{*}$

Gall bladder 710

Galvao, P. A. 429 *

Galvez, M. B. 458, * 792

Gamble, J. L., Jr. 432*

Ganapathy, C. 394*

Ganaway, R. L. 415*

Gane, R. 902*

Garcia, A. M. 404*

Garcia, Fuentes, M. 895*

Gardner, L. I. $363, * 369, * 389, * 393, *$ $441 *$

Garin, E. H. $424, * 455, * 460 *$

Garlinghouse, B. K. $465 *$

Garn, S. M. 345*

Garrison, R. D. 467 *

Garson, J. Z. 413*

Gartner, L. M. 364,* 366,* 404 *

Gaston, M. H. 420 *

Gaull, G. 432*

Gaull, G. E. 357 * $388 *$

Gautier, E. 894*

Gay, G. 398*

Gehler, J. 904,* 916 *

Geiger, H. 901*

Gelband, H. $350^{*}$

Gelfand, E. W. 397 *

Geller, G. 467*

Geller, G. R. 452*
Gender 200

Genetic disease 212, 553, 573, 598

Genetic disorder 865

Genton, N. 896*

Geoffroy, G. 392*

Gerald, P. S. 424*

Gerber, A. 905*

Gerbracht, L. 358*

Gerrard, J. W. 413*

Gersony, W. M. $355, * 363, * 467 *$

Gewitz, M. 353*

Giblett, E. R. 395*

Gigger, M. 451*

Gilbert, E. F. 439*

Gilbert, T. M. 809

Gilchrist, G.S. 360,* 398*

Gill, F. M. 411,*955

Gilles, F. H. 718

Gillette, P. C. $350^{*}$

Gimpert, E. 905*

Giorgio, A. J. 437*

Gitlin, D. 413,* 453*

Gitlin, J. D. 413*

Gitzelmann, R. $910^{*}$

Givens, J. R. 369*

Gjessing, L. R. 916*

Glade, P. R. 411*

Glader, B. E. 400 , $401 *$

Glasgow, A. 386*

Glasgow, A. M. 432*

Glasgow, L. A. 424, * 425 *

Glass, L. 446*

Gleason, W. A. 381*

Glew, R. 392*

Glezen, W. P. 425*

Glier, J. T. 212

Glode, M. P. 427*

Glomerular filtration 638

Glomerular filtration rate 758

Glorieux, F. H. 381,* 389,* 893,* $899 *$

Glovsky, M. 419*

Glucagon 37, 951

Gluck, L. $449, * 450 *$

Glueck, C. J. 434*

Glueck, H. I. 403*

Glucocorticoids 843

Glucose 238, 830

Glucose phosphate isomerase 18,26

Glucose Tm 638

Glutathione 852

Glycine 598, 721, 941

Glycine cleavage enzyme 721

Glycogen 238

Glycosphingolipids 89

$\mathrm{Gm}_{1}$ gangliosidosis, type I and type II 120

$\mathrm{GM}_{2}$ gangliosidosis 628

Gniot-Szulzycka, J. 388*

Godbole, V. 917*

Goetze, E. 357*

Goetze, H. 381*

Gold, E. 427*

Gold, R. J. M. 396*

Goldberg, H. I. 408*

Goldberg, M. D. 443*

Goldberg, S. 647

Goldberg, S. J. 342, *343,* $349, * 352 *$

Goldbloom, S. D. 351*

Goldblum, R. M. 401*

Golde, D. W. 407*

Goldis, G. 902*

Goldman, A. 375*
Golüman, A. S. $369, * 401, * 412 *$

Goldring, D. 371*

Goldsmith, D. I. $455^{*}$

Goldstein, A. S. 5

Goldstein, P. D. 381 *

Goldzier, S. 465*

Goluboff, N. 413*

Gonadal dysgenesis 740

Gonadotropins(s) 248, 740

Gonote, C. 417*

Good, R. A. $901^{*}$

Goodloe, D. 426*

Goodman, J. R. 413*

Goodman, S. I. 389,*396*

Gootman, N. 470*

Gordon, H. A. 914*

Gotlieb, A. 401*

Gotschlich, E. 427*

Gotschlich, E. C. 429*

Gould, J. B. 366*

Gould, L. V. 439*

Graham, J. R. 468*

Graham, T. P. 362*

Grand, R. J. 381,*765

Granger-Julien, M. 426*

Grant, J. A. 412*

Graw, R. G., Jr. 404*

Gray, M. E. 470*

Graziani, L. 462*

Grebin, B. 422*

Greener, Y. 358, * 434*

Greenwald, P. $390 *$

Greenwood, M. F. 401*

Greenwood, R. D. 350*

Gregory, T. 363,* 369*

Greifer, I. 454,* $459 *$

Grgic, A. 441*

Gribetz, D. 378*

Grieder, H. R. 909*

Griffith, J. F. 425,* 429*

Griffith, J. M. 352*

Grignon, B. 392*

Grignon, G. 393*

Grimes, E. R. 394*

Grimm, E. 377*

Griswold, W. 461*

Gross, E. 770, 774

Gross, G. P. 401*

Gross, I. 193

Grover, W. D. 390*

Growth 546, 783, 825, 960

Growth hormone 103

Growth retardation 109, 163

Growth transplantation 163

Growth velocity $149,157,929$

Grumbach, M. M. 373,*375*

Grupe, W. E. 457*

Gruskin, A. 344*

Gruskin, A. B. $455, * 561$

Guesry, P. 455*

Guignard, J. P. $894,{ }^{*} 896 *$

Guinee, V. $345^{*}$

Guller, B. $347,{ }^{*} 353 *$

Gunay, U. 401*

Gunn rat 647

Gur, A. 461*

Gursky, D. S. 377*

Gutberlet, R. L. 433*

Gutgesell, H. P. $350^{*}$

Gutman, L. T. 429*

Guyther, J. R. 573 
Habersang, R. 364*

Hack, M. 463*

Hacker, B. 455*

Hadorn, B. $384, * 899, * 905 *$

Hadorn, H. B. 381 *

Hagberg, S. 935

Hagenfeldt, L. 852

Haggins, J. C. $405, * 406 *$

Hagmann, R. 899*

Hahn, P. 37

Hales, B. $366^{*}$

Haljamäe, H. 899*

Hall, B. D. $390 * 4439 *$

Hall, D. J. $378^{*}$

Hallman, M. $447, * 874$

Halvorsen, K. 176

Halvorsen, S. 176

Hamberg, M. 908*

Hambidge, K. M. 386, 414*

Hamerton, J. L. 679

Hamilton, J. R. 382*

Hamilton, L. 443*

Hamilton, R. 354, * $379 *$

Hamilton, W. H. 433*

Hamly, C.-A. $386^{*}$

Hammersen, G. 389*

Hamwood, S. M. 740

Hananian, J. 410*

Handelsman, D. G. 357*

Hanefeld, F. 912*

Hansen, L. 879

Hansen, S. 433*

Hanson, L: A. 935

Hanson, V. 806

Harber, L. 437*

Hargrave, R. 417*

Harker, L. A. 416*

Harrah, J. 453*

Harris, H. 447*

Harris, M. B. 413*

Harris, S. $469, * 470^{*}$

Harris, T. R. $470^{*}$

Harris, V. 354*

Harrison, H. R. 431*

Hart, A. C. $436^{*}$

Hart, M. C. $422 *$

Harvey, D. R. $917 *$

Haruda, F. D. $380^{*}$

Harvey, M. A. 390*

Haslam, R. H. A. 363*

Hassanein, K. 960

Hastings, C. $371^{*}$

Hatahet, G. $439^{*}$

Hatcher, N. H. 390*

Hatemi, N. 903*

Hathaway, H. S. $402 *$

Hathaway, W. E. $401, * 402, * 408 *$

Hattler, B. G. 418*

Hattner, R. 458*

Hauhart, R. 238, 463*

Hawker, R. E. 349 , $^{*} 351 *$

Haworth, C. A. $899^{*}$

Haworth, J. C. $383, * 384 *$

Hayes, C. J. 363*

Haymovits, A. 438*

Hazlewood, C. F. 359*

Headings, V. E. $409 *$

Heald, F. P. $385^{*}$

Heart 67

Hedner, V. 789
Heggie, A. D. $425^{*}$

Heim, T. $357, * 911 *$

Helmke, K. $897^{*}$

Hein, L. 433*

Heiner, D. C. $419, * 421^{*}$

Heinonen, K. 913*

Heird, W. C. $381, * 384 *$

Helfer, R. E. $343^{*}$

Hellman, L. 378 , $380 *$

Helminths 652

Helson, L. 402*

Helwig, F. 471*

Hemoglobin 553

Hemoglobin Barts 955

Henderson, E. S. 404 *

Hendley, J. O. 428*

Hennekens, C. H. 350 *

Henning, S. J. 382*

Henriksson, P. 789

Henry, W. L. 352*

Hensen, S. A. $414,{ }^{*} 420 *$

Hepatic glycogenesis 114

Herbst, J. J. 379,* $382 *$

Herin, P. 758

Herod, L. 1

Herrmann, J. 440,* 441*

Hers, H. G. $910^{*}$

Herschkowitz, N. 904, * $908 *$

Herschkowitz, N. N. 865

Hess, R. O. $440^{*}$

Hessler, J. 467*

Heterokargon 606

Hexosaminidase A 628

Hexosaminidase B 628

Heymann, M. A. $351, * 352, * 354, * 451 *$

Hicks, R. M. 427*

Hicks, S. 440*

Hieber, V. C. $433 *$

Hill, D. E. $360^{*}$

Hill, H. R. 426 *

Hill, L. L. $456^{*}$

Hill, R. M. $363, * 369 *$

Hillman, L. S. 362*

Hillman, R. $362 *$

Hillman, R. E. 433,*941

Hiner, L. 455*

Hintz, R. L. $369^{*}$

Hipp, T. 344*

Hirose, F. M. $400^{*}$

Hirsch, J. A. $471^{*}$

Hirschfeld, S. $350^{*}$

Hirschhorn, K. 388,*390,*687

Hirshaut, Y. 417*

Hirvonen, L. 909*

Hitchcock, E. S. $344^{*}$

Hitzig, W. H. 905*

Ho, C. K. 433*

Ho, C. M. $347^{*}$

Hobel, C. J. $419 *$

Hochman, H. 438*

Hodes, D. S. 689

Hodes, M. E. 212

Hodgman, J. $444, * 451$ *

Hodgman, J. E. 257

Hoehn, H. 746

Hof, R. P. 451*

Hoffman, J. I. E. $352 *$

Hokegard, K. H. $912 *$

Holdredge, R. 343*

Holick, M. F. 454*
Holland, N. H. $370, * 426 *$

Holland, P. 401*

Hollerman, C. E. 458*

Holliday, M. 455*

Holliday, M. A. $109,378, * 453, * 458 *$

Hollingsworth, D. R. $369, * 435 *$

Holmes, L. B. $390 * 440 *$

Holmberg, C. $902 *$

Holoceruloplasmin 821

Holowach-Thurston, J. 238, 463*

Holsclaw, D. 353*

Holt, J. F. 444*

Holtzapple, P. 357 *

Holtzman, N. A. $382, * 821$

Hommes, F. A. $910 *$

Honig, G. R. $401^{*}$

Hood, M. W. 652

Hoogenraad, N. 361*

Hoogenraad, N. J. 5, 724

Hook, E. B. $344, * 390 *$

Hordof, A. 363*

Hordof, A. J. 467*

Horne, S. 413*

Hörnell, H. 852

Horning, M. G. $363, * 369 *$

Hougen, T. J. $463^{*}$

Housman, D. 387*

Houston, I. B. 894 *

Howald, B. 899*

Howard, J. B. 426*

Howe, W. R. $347 *$

Howell, R. R. $360, * 433 *$

Hrbek, A. 58, 912*

Hsieh, N. N. $456^{*}$

Hsu, L. Y. $390^{*}$

Hsu, S. L. $351^{*}$

Huang, N. N. $413, * 423 *$

Huang, T. L. $382, * 385 *$

Hubbard, D. W. 652

Hudson, R. L. 402*

Hue, L. 910*

Huehns, E. R. 398*

Huff, D. S. $413, * 420$ *

Hug, G. 430*

Hughes, E. R. 371*

Hughes, P. C. R. 149, 157

Hughes, W. T. $426, * 469, * 470 *$

Human growth hormone 710,800

Humbert, J. R. 414*

Hung, A. $369^{*}$

Hunt, C. E. $347, * 381, * 468,{ }^{*} 621$

Hunt, P. A. $344 *$

Hurtado, R. C. $414 *$

Hurwitz, R. $357^{*}$

Hustu, O. 409*

Hüther, W. 18

Hutzinger, O. 423*

Huxtable, R. F. 443*

Hyaline membrane disease 200

5-Hydroxyindolylacetic acid 809

$o$-Hydroxyphenylacetic acid 540

Hyman, A. I. 270

Hyman, B. H. 388*

Hyperammonemia 5

Hy perglycemia 721

Hyperlipidemia 114

Hyperphenylalanemia 704

Hyperuricemia 31

Hypopituitarism 800

Hypopituitary dwarfs 103 
Hypothermia 263

Hypoxanthine arabinoside 825

Hypoxanthine guanine phosphoribosyltransferase 31

Hypoxia 50, 58, 621

Iacuone, J. J. 417*

Iannetta, A. 422 *

I-cell disease 865

Idriss, Z. H. $430^{*}$

Ignatova, M. S. 914 *

Immunodeficiency Study Group 416*

Immunoglobulin(s) 806, 935

Immunoglobulin A 815

Immunoglobulin G. 815

Indermuhle, P. $897^{*}$

Infant 921

Ingall, D. 448*

Ingram, D. L. 429*

Inselman, L. S. 468*

Insulin 951

Intermittent positive pressure ventilation 792

Interstitial water 215

Intestine $673,765,884$

Intestinal parasite 652

Intrarenal blood flow 792

Irias, J. J. 433*

Ishikawa, T. 434*

Islet cells 951

Isoleucine 941

Isselbacher, K. J. 420*

Itabashi, H. 400*

Jabbour, J. T. 418*

Jackson, B. T. $348^{*}$

Jackson, C. E. $442 *$

Jackson, C. W. 357*

Jacobs, J. C. $426^{*}$

Jacobsen, J. 912*, 963

Jacobson, G. 383*

Jaffe, N. $350, * 402 *$

Jaffe, R. B. $371^{*}$

Jaiswal, V. 446*

Jakel, A. $894^{*}$

Jaksina, S. 765

James, H. L. 357 *

James, L. S. 450 * $454 *$

James, O. $456^{*}$

James, T. 370*

Jansen, G. R. 462*

Jansen, V. 796

Jansons, R. A. $365, * 372 *$

Jaszai, V. 911*

Jaundice 647

Jayatilaka, G. 445*

Jeejeebhoy, K. N. 379*

Jeschke, R. 897*

Jesse, M. J. $347, * 350$ 1*

Jilly, P. $385^{*}$

Jilly, P. N. 402*

Johanson, A. J. 371*

John, K. 405*

Johnson, A. 357 *

Johnson, D. G. 382*

Johnson, D. H. $350 * 351 * 355 *$

Johnson, E. A. $347 *$

Johnson, G. D. 806

Johnson, H. 402*

Johnson, J. D. 5, 431*
Johnsonbaugh, R. E. 369*

Johnston, M. V. 363*

Johnston, R. B., Jr. 411*

Jonasson, O. $417^{*}$

Joncas, J. H. 426*

Jones, E. L. $355^{*}$

Jones, E. M. 238, 46ः*

Jones, H. W. $372 *$

Jones, J. M. $415^{*}$

Jones, K. L. $390, * 440$ *

Jorgensen, H. 400*

Jose, P. $458^{*}$

Jose, P. A. 456*

Joshi, V. V. 415*

Josso, N. 755, 968

Jouja, V. $910^{*}$

Juan, C. S. $367 *$

Jung, A. L. $379^{*}$

Jungas, R. L. $438^{*}$

Jusko, W. J. 358 * 375 *

Kachra, Z. 396*

Kaffe, S. 411,* 414*

Kaijser, B. 935

Kaiser, D. $905^{*}$

Kaitila, I. I. $440^{*}$

Kalafaic, Z. 915*

Kalavsky, S. $405^{*}$

Kalhan, S. $370^{*}$

Kalinowsky, W. 18

Kallen, R. J. 457*

Kamensky, E. 393*

Kandall, S. R. 364*

Kanfer, J. N. 392,* 546

Kanra, G. Y. 420*

Kanto, W. P., Jr. 447*

Kaplan, B. S. $348^{*}$

Kaplan, D. 394*

Kaplan, G. W. $459^{*}$

Kaplan, J. 402,* 408, ${ }^{*} 414 *$

Kaplan, R. 381*

Kaplan, S. $347, * 350 *$

Kaplan, S. A. 244, 362*

Kaplan, S. L. $344, * 373, * 375 *$

Kapoor, S. 348*

Karatela, S. A. $419 *$

Karelitz, S. 921

Karlsson, K. 50, 58, 912*

Karp, L. E. 746

Kashgarian, M. 461*

Kass, E. H. 428*

Kastner, H. 82

Katona, P. 468*

Katona, P. G. 351*

Kattwinkel, J. 468*

Katz, L. 382*

Katz, R. M. $416^{*}$

Kauffman, R. E. 364*

Kaufman, R. L. 439*

Kaufman, S. 714

Kawahara, F. S. 434*

Kawai, N. 353*

Kayden, H. J. 796

Kaye, C. I. 120

Kazazian, H. H., Jr. 403*

Kazmaier, K. 463*

Keane, J. F. $349,{ }^{*} 351 *$

Keating, J. P. $381 * 407 *$

Keenan, B. S. $372 *$

Keenan, S. B. $375^{*}$
Keenan, W. J. 445,* 463*

Keith, R. W. 447*

Kelch, R. P. $370^{*}$

Keller, A. $346^{*}$

Kelley, J. L. 412*

Kelley, V. C. $375^{*}$

Kelly, D. 352*

Kelly, D. T. 352*

Kempin, S. J. 357*

Kennedy, J. L., Jr. 447*

Kennel, J. 345*

Kenny, F. M. 367,* $373,{ }^{*} 408 *$

Kenny, J. F. 411,* 414*

Kerber, I. J. $369^{*}$

Kern, K. A. 594

Kerr, D. S. 434*

Kersey, J. H. 403*

Kessler, D. L. $430^{*}$

Ketotic hyperglycemia 721

Khachadurian, A. K. 386,* 434 *

Khalifa, A. S. 403*

Khalil, M. 387*

Khan, A. J. $463^{*}$

Khan, M. A. $385^{*}$

Khaw, K. T. $350, * 351, * 355, * 430 *$

Khoury, G. H. 351 *

Kidney(s) 109, 546, 561, 638, 659, 673, $735,758,792,935$

Kiely, B. 352*

Kienstra, R. A. $456^{*}$

Kiernat, J. F. 399*

Kim, H. . . 390*

Kim, H. W. $415, * 422 *$

Kimura, R. E. $361^{*}$

Kimzey, S. L. 359*

King, B. D. $441 *$

King, M. T. $441 *$

Kinky hair syndrome 821

Kinsbourne, M. 353*

Kirby, L. 37

Kirkland, R. 371 *

Kirkland, R. T. $370,{ }^{*} 376,{ }^{*} 377 *$

Kirkpatrick, S. E. $351 *$

Kisker, C. T. $403^{*}$

Kitterman, J. A. 358,* 447 *

Kjellmer, I. 50, 58, $912 *$

Klagsbrun, M. 205

Klain, D. B. 464 *

Klatskin, E. $405^{*}$

Klaus, M. $345, * 463, * 468^{*}$

Kleihauer, E. $900^{*}$

Klein, P. 366*

Klein, P. S. 344*

Kleinman, L. I. $451, * 456 *$

Klingberg, W. G. 351*

Klish, W. 388*

Klish, W. J. $382, * 835 *$

Knight, E. A. 446*

Kniker, W. T. $415^{*}$

Knoller, M. 394*

Knufermann, H. 900*

Koch, H. H. 18

Kochen, J. 358,* 403,* 434,* $460 *$

Kochen, W. 906*

Koenig, H. M. 404*

Kogut, M. D. $367, * 368^{*}$

Kohaut, E. C. $456^{*}$

Kohlschuetter, A. 908*

Koldovsky, O. 357*

Kolotkin, M. 103 
Komazawa, M. 404*

Komp, D. M. 75

Kontras, S. B. $404, * 441 *$

Korelitz, B. I. 378*

Kornreich, H. K. 806

Korsch, B. M. $343, * 458 *$

Korth-Schutz, S. 163

Koskimies, O. 453*

Koss, M. N. 461*

Kotas, R. V. 364 *

Kousseff, B. G. $390^{*}$

Kraft, S. C. $418^{*}$

Krantz, M. 382*

Krasner, J. 364*

Krassner, L. S. $461 *$

Krauss, A. N. $452, * 464 *$

Kream, J. 380*

Krebs, H. M. 418*

Krebs-Henseleit cycle 5

Krempien, B. 896*

Kretchmer, N. $357, * 361, * 382, * 724$

Krieger, I. 358*

Krohn, H. P. 893*

Kroll, W. A. $390^{*}$

Kron, R. E. 344 , $364 *$

Kronemer, N. S. $371 *$

Kronenberg, L. H. 358*

Krouskop, R. W. $383, * 444, * 448 *$

Krovetz, L. J. 349,*351*

Kuhns, L. R. 444*

Kuitunen, P. 440*

Kulin, H. E. 740

Kumar, M. L. 427*

Kumin, S. 437*

Kwateng, K. $367^{*}$

Labor 1

Lachman, R. 354*

Lacourt, G. $916^{*}$

Lakier, J. B. $351 *$

Lala, R. V. 446*

Lampert, P. W. 825

Lancaster, G. A. 358*

Landau, L. I. 468*

Lanman, J. T. 1, 448*

Lansky, L. 364 *

Lanzkowsky, P. 396*

Laraya-Cuasay, L. 413*

Laraya-Cuasay, L. R. 423*

Larsen, P. R. 373*

Larson, E. 427*

Larsson, A. 852

Larter, W. 352*

Lasalle, R. 385*

Lassila, E. $359, * 361 *$

Laster, L. 459*

Latt, S. A. $391^{*}$

Lau, S. S. $369^{*}$

Lauersen, N. 366*

Laupus, W. E. $415^{*}$

Lavin, N. 362*

Lazarus, M. $457 *$

Leake, R. D. 434, * 457 *

Lebenthal, E. 383*

Leboeuf, G. $368^{*}$

Lebowitz, J. 437*

Lecithin 848,874

Leckie, M. P. 463*

Lee, C. K. $408^{*}$

Lee, C. L. Y. $391^{*}$
Lee, E. M. 815

Lee, J. J. 431*

Lee, K.-s. 404*

Lee, P. A. $371^{*}$

Lee, S. K. 457*

Lee, T. C. $433 *$

Lee, Y. C. $382 *$

Lee-Foon, W. $471 *$

Leffert, F. 365*

Leidy, G. $422, * 429 *$

Leikin, S. L. $415^{*}$

Lenard, H. G. 907*

Leonard, A. S. $381, * 468, * 621$

Lepage, G. $385^{*}$

Lepow, M. L. 437*

Lesch-Nyhan syndrome 31

Lester, R. 366*

Letarte, J. 368*

Levan, N. 257

Leventhal, B. G. 404*

Levey, R. 457*

Levin, B. 857

Levin, D. L. $355^{*}$

Levine, A. S. 404*

Levine, L. S. $352 * 372 * 374 *$

Levine, M. D. $471^{*}$

Levine, M. R. 606

Levine, O. R. 270

Levine, R. L. 724

Levison, H. 349 ,* 466*

Leviton, A. 718

Levitz, M. 796

Levkoff, A. 456*

Levy, G. $365^{*}$

Levy, H. L. 389,* 391,* 434*

Lewis, A. B. $352 * 354 *$

Lewis, R. M. $355^{*}$

Lewy, J. E. 163, 352,* 453*

Lewy, P. R. 457*

L'Heureux, P. 381 *

Li, I. O. W. $365^{*}$

Liappis, N. 894*

Lieberman, E. 109

Lietman, P. S. 363*

Lieu, S. $470^{*}$

Lievaart, P. 411 *

Lifshitz, F. 109, 380,* 383,* 735

Lightsey, A. L., Jr. 404 *

Lilien, D. 402*

Lilienfield, L. S. 456*

Liljestrand, J. D. 404*

Lim, W. 417 *

Lin, H.-J. $375^{*}$

Lin, S. 457*

Linarelli, L. $373, * 467 *$

Linarelli, L. G. $435^{*}$

Linblad, B. S. 916*

Lind, J. 912*

Lindemann, R. 916*

Link, E. 897*

Linoleic acid 82, 263

Linolenic acid 263

Linshaw, M. $344^{*}$

Lipid metabolism 13

Lipids 93

Lipids, brain 783

Lipolysis 800

Lippe, B. 435*

Lipson, E. H. 441*

Lipson, M. 434*
Lischner, H. W. 413,*423*

Litt, I. F. 392*

Litt, M. $344, * 364$ *

Liver 37, 193, 714, 765

Liu, H. H. $382 *$

Lloyd, J. K. 911 *

Lockhart, E. A. 457*

Loda, F. A. 425*

Lodinova, R. 910*

Loew, A. D. $468^{*}$

Loirat, C. $901^{*}$

Lombeck, I. 82, 904*

Lombroso, C. $435^{*}$

Lomola, A. 437*

Londe, S. 371*

Long, W. K. 430 *

Lonsdale, D. $432, * 435 *$

Lopez, R. 379*

Lorand, L. 789

Lorch, V. 378,* 405*

Lorentz, W. B. 454*

Lott, I. T. 434*

Lovecchio, J. 364*

Lovejoy, F. H. 435, * 438*

Lowden, J. A. 387*

Lowrie, E. 457*

Lu, C. $367 *$

Lucas, R. V., Jr. 347*

Luckasen, J. R. 403*

Lull, R. J. 433*

Lund, H. T. 912*

Lundgren, D. W. 446*

Lung 193, 200, 244, 270, 848, 874

Lupu, A. N. 611

Lusher, J. 403*

Luteinizing hormone 740

Luthi, C. 899*

Lutz, P. $906^{*}$

Lutz, R. W. 458*

Lux, S. E. $387 * 405 *$

Lyles, R. L., Jr. 75

Lymph 270

Lynch, V. A. 449*

Lysine 42

Lynfield, J. 352*

Lynn, H. B. $398^{*}$

Lyons, E. C. $458, * 459 *$

Lysine- $\alpha$-oxoglutarate reductase 42

Lysolecithin 874

Lysosome(s) 82,774

Ma, R. 383*

Mabry, C. C. $435^{*}$

Macdonald, H. 434*

MacDonald, H. M. 443*

MacDonald, M. J. 431*

MacGillivray, M. H. 103, 371*

Macken, J. $902 *$

MacKenzie, J. W. 413*

Macklem, P. T. 468*

Maclaren, N. K. $391 * 435 *$

MacLeod, S. M. 362*

Macmillan, R. 381*

MacNamara, H. 405*

Macpherson, G. A. 806

Maddaiah, V. T. $371, * 377^{*}$

Madore, C. $449 *$

Magalnick, H. $460^{*}$

Magruder, C. S. $365^{*}$

Mahoney, M. J. 387 ,* 436,* $437 *$ 
Main, C. A. $409 *$

Makker, S. 458*

Makowski, E. L. 830

Makres, T. D. 426*

Malas, K. 463*

Malekzadeh, M. H. 458*

Malnutrition 149, 157, 200

Malone, J. I. 436*

Maloney, J. D. 353*

Maltz, H. E. 458*

Malvaux, P. 800

Mameesh, M. 383*

Mamunes, P. $344, * 436 *$

Maningas, C. S. $413^{*}$

Manring, A. 347*

Manz, F. 894*

Mann, M. D. 879

Mapa, H. C. $394^{*}$

Mardini, M. K. $352 *$

Margolis, C. Z. $472 *$

Markey, S. P. 389*

Markovs, M. E. $370^{*}$

Marks, M. I. 427*

Marliss, E. B. 438*

Maron, B. J. 352*

Mars, H. 468*

Marshall, R. N. 376*

Martens, R. J. 64

Martin, G. M. 746

Martin, H. L. 421*

Martin, J. M. 438*

Martin, R. 470*

Martinez, B. 414*

Martinez, G. 405*

Martinez, J. 415*

Martinez, M. 89, 93

Massa, E. 408*

Mastromarino, A. J. 385*

Matalon, R. 436*

Matalon, S. $468, * 621$

Maternal starvation 890

Mathéová, E. 914, ${ }^{*} 917$ *

Matsuda, I. 821

Mattar, M. E. 358*

Mattheis, M. J. $422 *$

Matthews, L. W. $422, * 465, * 861$

Maturation defect 591

Maurer, H. M. 405, * 406*

Max, S. R. 391*

Mayo, J. W. 861

Mayor, G. 896*

McAdams, A. J. $461^{*}$

McBean, L. D. 837

McCauley, E. 344*

McCormick, A. Q. 448*

McCoy, E. E. $395^{*}$

McCracken, G. H. 426,* 427,* 428*

McCrea Curnen, M. G. 342*

McCue, C. M. 406*

McCulley, L. B. 363*

McEwan, S. N. 469*

McInnes, R. R. $391 *$

McIntire, K. R. $407 *$

McIntire, M. S. $397 *$

McIntosh, K. 422,* 689

McIntosh, N. 896*

McIntosh, R. M. 461*

McIntosh, S. 405, * 406*

McKenna, B. A. $459^{*}$

McKey, R. M. $386^{*}$
McLaughlin, B. 426*

McLaughlin, G. W. $380^{*}$

McMaster, J. H. 408*

McNabb, P. C. $426^{*}$

McNamara, D. G. $350^{*}$

McNatt, M. L. 371*

McVicar, M. 380*

McWilliams, N. B. $405, * 415 *$

Mehes, K. 898*

Mehls, O. $894, * 896, * 913 *$

Mela, L. $356^{*}$

Melançon, S. B. 388,* 392*

Melish, M. E. $427^{*}$

Mellins, R. B. $467,{ }^{*} 468 *$

Mellman, W. 442*

Melsher, H. L. 432*

Menano, H. P. $900 *$

Mendoza, S. A. 459*

Menezes, J. 426*

Menkes, J. H. 263, 783

Merchant, K. 433*

Merida, M. A. 414*

Merritt, A. D. 212

Mescher, E. J. 358*

Meschia, G. $360, * 830$

Mesrobian, A. 353*

Metcoff, J. 383*

Metabolic acidosis 659

Metabolic homeostasis 659

L-Methionine 673

Methyl arachidonate 1

Methylation 205

Methylation of RNA 212

Methyl linoleate 1

Meuwissen, H. J. 406,* $415^{*}$

Meyer, H. B. P. $445^{*}$

Meyer, R. A. $347, * 350 *$

Meyer, W. J. 372*

Meyer-Bahlburg, H. F. L. 344*

Middelton, P. 379*

Midgley, A. R., Jr. 371 *

Migeon, C. J. $371,{ }^{*} 372 *$

Milic-Emili, J. 467,* 471 *

Miller, C. D. $436^{*}$

Miller, G. 428*

Miller, H. 353*

Miller, H. C. 960

Miller, J. 394*

Miller, J. W. 466*

Miller, L. D. $445^{*}$

Miller, M. $410^{*}$

Miller, M. E. 406, * 415*

Milner, R. D. G. $908,{ }^{*} 917^{*}$.

Milstein, M. 392*

Milunsky, A. 391,*392*

Mims, L. C. $364^{*}$

Miranda, S. 463*

Mirkin, B. L. 461 *

Mitchell, A. $400^{*}$

Mize, C. E. $384^{*}$

Mizrahi, L. 359*

Mockrin, L. $347, * 448, * 468 *$

Modell, C. B. $398^{*}$

Modler, S. $467 *$

Moe, P. $389 *$

Moffet, C. L. 345*

Mohyuddin, F. 358,* 391 *

Monedjikova, V. 424*

Monkus, E. $365, * 429, * 436$ *

Monn, E. 903*
Monnens, L. A. H. 598, 896*

Monroe, G. A. 349*

Montgomery, S. 456*

Moore, E. C. $406,{ }^{*} 415 *$

Moore, E. S. $449, * 458, * 459, * 792$

Moore, L. $414^{*}$

Moore, W. T. 400*

Morag, A. 415*

Morag, B. 415*

Morecki, R. 424*

Morgan, N. F. 363*

Morgan, P. M. 652

Morin, C. C. $893^{*}$

Morin, C. L. $381, * 385 *$

Morishima, A. 392*

Morrell, R. E. 427*

Morris, J. A. $364^{*}$

Morrison, J. 434*

Morriss, F. H. $360^{*}$

Morrow, G. 436, * 437 *

Mortimer, E. A., Jr. 472*

Mosaicism 679

Moshang, T., Jr. 372*

Mosier, H. D., Jr. 365 * $372 *$

Mosovich, L. 358*

Mrozinska, K. S. $443^{*}$

Mucosa 82

Mukhopadhyay, N. $400^{*}$

Müller, M. 704

Müllerian ducts 968

Müllerian-inhibiting activity 755

Munschauer, R. W. 103

Muranyi, L. 900*

Murib, A. 909*

Murphy, J. V. 392,*393*

Murphy, M. L. $345,{ }^{*} 408 *$

Murray, K. 373*

Murrer, A. $425^{*}$

Murrmann, S. K. $411^{*}$

Mürset, G. 248

Muscie 257

Mushin, A. S. 908*

Mutation 120

Myerburg, R. J. 350 *

Myers, K. 406*

Myrianthopoulos, N. C. $440^{*}$

Nadas, A. S. $350^{*}$

Nader, P. R. $344^{*}$

Nadler, H. L. $120,170,387, * 388,{ }^{*} 395, *$ 684

Naeye, R. L. 200

Nahmias, A. J. $419, * 423 *$

Nahmias, J. 345*

Nakagawa, S. $357, * 437 *$

Nakamura, E. $387 *$

Nakamura, F. T. 387*

Nakatani, S. $361, * 393 *$

Naliboff, J. $351 *$

Nanavati, A. $417^{*}$

Nankervis, G. A. 427 *

Nash, M. A. $453, * 459 *$

Nash, M. A. 638

Nathan, D. G. $398, * 471, * 553$

Nathenson, G. 405*

Navojosky, B. 345*

Neal, W. A. $347, * 468 *$

Nearman, H. 468*

Necheles, T. F. 421*

Neiburger, R. G. $416,{ }^{*} 417 *$ 
Neiderer, B. $442 *$

Neims, A. 366*

Nelson, J. D. $428 *$

Nelson, R. D. $416^{*}$

Nelson, R. J. $354^{*}$

Nelson, T. 963

Neonate $31,89,200,215,244,257,621$, $647,714,789,821,825,843,852,884$, 955,960

Nephrogenic diabetes insipidus 223

Nergardh, A. $912 *$

Nervez, C. T. 437 * $453 *$

Nesbit, M. E. 403*

Neu, R. L. $393, * 441 *$

Neuburger, N. 466*

New, M. 366*

New, M. I. $163,352, * 372,{ }^{*} 374 *$

Newberger, E. H. 345*

Newhouse, M. T. $469^{*}$

Nichols, B. L. $359 * 382 * 385$ * $388 *$

Niethammer, D. $900, * 901 *$

Niewczas-Late, V. 679

Nigam, S. K. $446^{*}$

Nilsson, I. M. 789

Nilsson, U. R. 459*

Nitowsky, H. M. 357,* $395, * 397, * 437$ *

Nkongo, A. 424 *

Noaketotic hyperglycinemia 598

Noonan, J. A. 353*

Norberg, W. $467 *$

Norepinephrine 37,809

Norman, M. E. 459*

Northway, J. D. $368^{*}$

Norton, J. B., Jr. 353*

Noto, T. $405^{*}$

Novak, M. 365*

Nowlin, J. 363*

Nugent, E. W. $352 *$

Nussle, D. $897^{*}$

Nutrition 960

Nyhan, W. L. 31

Oakes, G. K. 446*

Oates, R. K. $917^{*}$

Oberholzer, V. G. 857

O'Brien, D. 431*

O'Brien, J. F. 904*

O'Brien, R. T. $405, * 406 *$

Ocampo, M. $458 * * 459 *$

Oche, H. D. $413^{*}$

Ochs, H. D. $416^{*}$

O'Connell, K. C. $470^{*}$

O'Connor, J. F. 448*

O'Croinin, P. 404*

Oda, A. I. $430^{*}$

Odell, G. B. $399, * 407 *$

Odell, J. L. 399*

Odell, W. D. $368^{*}$

Oetliker, O. 899*

Ogra, P. L. $415, * 416^{*}$

Oh, W. $354, * 356, * 359, * 374, * 432, *$

$434, * 439, * 457, * 468, * 783,951$

Oi, V. T. $412 *$

Okoroma, E. O. 353*

Olambiwonnu, N. O. $372, * 407 *$

Olbing, H. 459*

Oldham, S. B. 454*

Olling, S. 935

Olsson, T. 50, 58
Olver, R. E. $466^{*}$

O'Mara, R. E. 402*

Omdal, C. $343^{*}$

Omori, K. 783

O'Neal, P. L. 406*

Opitz, J. M. $440^{*}$

Oppenheimer, E. H. 384*

O'Quin, R. 431*

Ordơnez, J. V. 385*

O'Reilly, R. 416*

Ornithine decarboxylase 231

Ornithine transcarbamylase 5

Orotic aciduria 5

Orr, I. A. 689

Orskov, F. 427,* $428 *$

Orskov, I. $154,427, * 428 *$

Osborne, R. G. 437*

Osher, A. B. 594

Oski, B. F. $407^{*}$

Oski, F. A. $64,346, * 404, * 406, * 407, *$

$410, * 420, * 439 *$

Osler, D. C. $359^{*}$

Osnes, J. B. $903 *$

Osserman, E. F. 418*

Ostrea, E. M., Jr. $365, * 449 *$

Oho, E. F. 941

Overall, J. C., Jr. $427 *$

Owen, G. M. 345*

Owens, K. E. 396*

Oxman, M. N. 430*

Oxytocin 1

Oye, I. 903*

Ozand, P. 433,* 435*

Ozere, R. L. 423*

Pabico, R. C. $459 *$

Pabst, H. F. 395, * 416 *

Pachman, L. M. 407,* $417 *$

Pagel, C. J. 399*

Pagliara, A. 407*

Pahuja, J. N. $371, * 377$ *

Pai, K. G. 435 *

Pain sensitivity 921

Pallavicini, J. C. 531

Palmer, J. 413*

Palmer, T. 857

Paneth cells 82

Pangburn, P. C. $414 *$

Panner, B. 459 *

Pantelakis, S. N. 917*

Papadopoulou, Z. 458*

Papageorgiou, P. S. 411,* 414*

Papile, L.-A. 444*

Pardo, V. 436,* 461*

Park, C. D. $348^{*}$

Park, E. 452*

Park, J. I. 372*

Park, S. 468*

Parkhill, W. S. 377*

Parkman, P. 417*

Parks, J. S. $133,370, * 371, * 372, * 376, *$ $377, * 407 *$

Parrott, R. H. 148, 415,*422*

Parsons, B. . 434*

Partin, J! C. 378 * 384 *

Partin, J.S. $384 *$

Passey, R. B. 454*

Pasternak, A. 902*

Paton, J. B. $386, * 449, * 458, * 459, * 792$
Pattison, C. P. 422 *

Paul, B. 394 *

Paul, M. H. $355^{*}$

Paunier, L. 898 * $908 *$

Pavan, M. 606

Pavkovcekova, O. 914,*917*

Payne, R. 388*

P-creatine 238

Pearson, H. A. 406*

Peason, T. 821

Peckham, G. J. 348*

Pellié, C. 540

Peltonen, R. 909*

Peltonen, T. 909*

Penn-Walker, D. $365^{*}$

Penny, R. 372,* 407 *

Pereira, W. 446*

Pergolizzi, R. 383*

Perheentupa, J. 902,* 904 *

Perheentupa, J. I. 440*

Perlstein, P. H. $445^{*}$

Perricelli, A. $453^{*}$

Perry, T. L. 433*

Petersen, I. 912*

Petersen, N. J. 422*

Peterson, B. 453*

Peterson, G. E. $375^{*}$

Peterson, R. E. 372*

Peterson, W. D. 414*

Petigrow, C. S. $411^{*}$

Pettersen, J. C. $439 *$

pH 50, 963

Phang, J. M. 396*

Phelps, D. L. $356, * 359, * 468 *$

Phenylalanine 540

L-Phenylalanine 704

Phenylalanine hydroxylase 714

Phenylketonuria 540, 704, 809

Phenylpyruvic acid 540

Philippart, M. 359, *361,*393*

Phillips, P. $417^{*}$

Phoenix, M. D. 344*

Phosphoenolpyruvate carboxykinase 37

Phospholipids 1, 710

Phosphoribosyl pyrophosphate 31

Phototherapy 257, 573, 647

Phyliky, R. L. 433*

Piasecki, G. J. 348*

Piazolo, P. $897^{*}$

Picou, D. 434*

Pieroni, D. R. $347 *$

Pildes, R: 354 *

Pildes, R. S. 437, * 443*

Pinkel, D. 409*

Pinsky, L. 394*

Piomelli, S. $345, * 398, * 437 *$

Pippenger, C. E. $362, * 366 *$

Pirie, G. E. 448*

Pitt, J. 384*

Placenta 697, 770, 774, 796

Platzker, A. C. G. 358*

Plauth, W. H. $355^{*}$

Plotkin, S. A. $417^{*}$

Pluess, H. J. $905 *$

Plumer, L. B. $459^{*}$

Poh-Fitzpatrick, M. 437*

Poirier, R. $381, * 893 *$

Poland, R. L. 407*

Poley, J. R. 710

Polgar, G. 465* 
Polin, R. A. 384 *

Pollack, P. F. 384*

Polmar, S. H. 417*

Polonovski, C. 857

Polyamines 231

Pomerance, J. J. 449, * 450, * 469*

Poon, K. A. 377*

Popovic-Rolovic, M. 914*

Porter, I. H. 394*

Postheparin lipoprotein lipase 114

Potier, M. 388, $392 *$

Potter, E. 417*

Potts, E. 382*

Powell, K. R. 463*

Powers, W. F. 449,* 469*

Poznanski, A. K. 444 *

Prader, A. 248

Prandota, J. 365*

Prasad, A. 384*

Precocious puberty 248

Prednisolone 37

Premature infant(s) $257,263,591,659$

Prenatal diagnosis 679, 746

Preston, D. $370^{*}$

Preus, M. 441*

Pribylova, H. 436*

Price, R. A. 469*

Priest, J. H. 431 *

Prince, P. E. 344*

Proctor, K. G. 443*

Proesmans, W. 902*

Protein synthesis 770

Protozoa 652

Provisor, A. J. 417*

Pruitt, A. W. 365*

Pryles, C. V. 463*

Przyrembel, H. 904*

Puck, M. 394*

Pullon, P. A. 439 *

Putrescine 231

Pyroglutamic aciduria 852

Pyelonephritis 935

Qazi, Q. H. 394*

Quan, L. 390*

Quartner, J. 433*

Quie, P. G. 426*

Quinn, P. 464*

Rachelefsky, G. 420*

Rachelefsky, G. S. 417*

Radde, I. C. $452^{*}$

Radel, E. 403,* 460*

Radfar, N. 373*

Radvany, R. 388*

Rafuse, E. V. 377*

Ragab, A. H. $407 *$

Rahbar, F. 385,* 450*

Rai, U. S. 421*

Räihä, N. C. R. 357 * 911,*913*

Raivio, K. $447, * 874$

Rall, T. W. 911*

Ramakrishnan, R. 609

Rampini, S. 704

Randall, J. E. 428*

Rao, G. H. R. 421 *

Rao, G. J. S. 684

Rao, I. P. 451*

Rapoport, J. 464 *

Rappaport, H. 349*
Rashkind, W. J. 348*

Rathbun, M. A. 442*

Ratner, H. 348*

Ravitch, M. M. 380*

Rawson, J. E. 462*

Ray, C. G. 423*

Raye, J. R. 437 *

Redman, W. 429*

Reed, M. E. 423*

Reibman, J. 398*

Reid, R. H. $418^{*}$

Reimold, E. W. 460*

Reins, J. 417*

Reiser, S. 884

Reiss, J. 465*

Reiter, E. O. $373, * 740$

Reivich, M. 408*

Renal acidosis 735

Renal transplantation 163

Renin 611

Rennert, O. M. $905^{*}$

Reo, F. 540

Renton, K. W. 362*

Repice, J. P. 373*

Respiratory distress syndrome 621,689

Retina 945

Rey, H. $450^{*}$

Rey, J. 540

Reynolds, D. W. 428,*430*

Reynolds, J. W. 452*

Rezvani, I. 373*

Ribosomal RNA 205

Ribosomes 770

Richard, G. A. $424, * 455$, 460 *

Richards, B. $917^{*}$

Richards, C. 373*

Richardson, C. J. $450, * 469 *$

Richmond, J. B. $345^{*}$

Ricour, C. $897, * 903 *$

Ridge, S. C. 870

Rieger, C. H. L. $418^{*}$

Riffell, M. I. 679

Rifkind, A. 366*

Rigatto, H. 450*

Riggio, R. R. 163

Riha, M. 58

Ringler, N. 345*

Ripley, B. A. 628

Ritz, E: 896,* $913 *$

Rivers, R. P. A. 408*

Roan, Y. 386,* 459*

Robbins, F. C. 972

Robbins, J. B. $427, * 428 *$

Roberson, H. 359*

Robertson, B. 789

Robertson, L. W. 406*

Robillard, J. E. $460^{*}$

Robinson, A. 394,* 403*

Robinson, H. M. 434*

Robson, A. M. 456, ${ }^{*} 460 *$

Rodriguez, J. R. 385*

Rodriguez, J. T. 382,* $385 *$

Rodriguez Soriano, J. 895*

Roe, E. J. 471*

Roeder, L. M. 385 *

Rogers, B. L. 431 *

Rogers, J. H., Jr. 380 *

Rogers, S. 379*

Rogol, A. D. 374*

Rogolsky, M. 425*
Rola-Pleszczynski, M. 414*

Rolfe, U. T. 422*

Roloff, D. W. 447*

Root, A. 375*

Root, A. W. $374,{ }^{*} 455 *$

Root, R. K. 413*

Rosan, R. C. $469^{*}$

Rose, A. L. 366*

Rosen, G. 408*

Rosen, J. F. 438*

Rosén, K. G. 50, $912 *$

Rosen, S. 374*

Rosen, T. S. 366*

Rosenberg, L. E. 436*

Rosenbloom, A. L. 441*

Rosenfeld, W. N. 446,* $450 *$

Rosenfield, R. L. 374*

Rosengart, R. 354*

Rosenquist, G. C. 353 *

Rosensweig, N. S. 381*

Rosenthal, A. $349, * 350, * 351, * 353,{ }^{*}$ $355^{*}$

Rosenthal, M. D. $361^{*}$

Rosner, B. 428*

Ross, J. 466*

Rossman, C. M. 469*

Rosso, P. 359*

Rothberg, R. M. 418*

Rowe, M. 468*

Rowe, R. D. $347^{*}$

Roy, C. C. $385^{*}$

Roy, N. R. 452*

Roy, R. N. 379*

Rozee, K. R. 423*

Rubin, A. L. 163

Rubin, H. 435*

Rucknagel, D. L. 388*

Rudiger, H. W. 906*

Rudloff, H. B. $401 *$

Rudnik, M. 348*

Rudolph, A. M. $351, * 352, * 354, * 451 *$

Rudoy, R. 428*

Ruley, E. J. $460^{*}$

Rumpelt, H. J. 902*

Rupert, G. 456*

Rush, D. $346, * 450^{*}$

Russell, M. O. 408*

Rutzky, J. 409*

Ryness, J. 903*

Saadat, M. $385^{*}$

Sabin, A. B. 978

Sabio, H. $360^{*}$

Saccharopine 42

Saccharopine dehydrogenase 42

Saccharopine oxidoreductase 42

Sack, J. 374*

Sacktor, B. 454*

Sadeghi-Nejad, A. $374, * 438 *$

Saenger, P. 163

Saenger, P. H. 372 , * 374 *

Saffer, S. I. $384^{*}$

Sahn, D. J. $354^{*}$

Salafsky, I. S. 342*

Saliva 531,861

Sallan, S. 402*

Salmon, S. E. 418 * $419 *$

Salzberg, A. M. 378*

Samuelsson, B. 908*

Sandberg, D. H. 386* 
Sandhoff's disease 628

Sandler, P. 458*

Santamarina, B. 553

Santulli, T. V. $384 *$

Sanyal, S. K. 196, 496,* 470*

Sapire, D. $455^{*}$

Sarff, L. D. $427, * 428 *$

Sarkozi, L. 446, * 450*

Sarkozy, E. 399*

Saudubray, J. M. 857

Saunders, E. F. 397*

Savage, D. C. L. 373*

Savilahti, E. $440^{*}$

Sbirakis, S. 917 *

Schade, C. P. $345 *$

Schaffner, F. 432*

Schaffner, T. 909*

Schaller, J. 423*

Schanberger, J. E. 422*

Scharschmidt, B. F. 573

Schay, M. I. 765

Schenck, C. 344*

Schenk, H. 357*

Scherzer, A. 453*

Schiff, D. 398*

Schiffer, M. 427*

Schilder's disease 170

Schindler, S. 355*

Schleman, M. M. 470*

Schlueter, M. A. 354 , $447 *$

Schmickel, R. D. $394 * 433 *$

Schmidt, R. 395*

Schnall, B. S. 441 *

Schnaufer; L. $407^{*}$

Schneider, A. 899*

Schneider, J. A. $390^{*}$

Schneider, P. 898*

Schneider, T. R. 407*

Schoenman, M. 735

Schretlen, E. D. A. M. 598

Schröter, W. 18, $900 *$

Schubert, W. K. $378, * 384, * 430 *$

Schuit, K. E. $360^{*}$

Schuler, D. 915*

Schulkind, M. L. 418*

Schulman, H. M. 400 *

Schuman, H. 449*

Schuster, E. 915*

Schut, J. 363*

Schwabe, U. 893*

Schwartz, A. 355*

Schwartz, A. D. $355, * 407 *$

Schwartz, A. L. 911 *

Schwartz, E. 163, 374,* 408,* 955

Schwartz, R. 370*

Schwartz, R. H. 594

Scleroderma 806

Scott, C. R. $395^{*}$

Scott, M. D. $375^{*}$

Scott, R. B. $385, * 402, * 410 *$

Scott-Emuakpor, A. B. 858

Scranton, P. E. $408^{*}$

Scriver, C. R. $358, * 389, * 391, * 899 *$

Scrutton, M. C. $390^{*}$

Seaman, C. $398 * 437 *$

Seeds, J. M. 369*

Seely, J. R. 710

Segal, D. J. $395 *$

Segal, S. $379 * 448 *$

Segel, G. B. 594
Seip, M. 916*

Selinger, S. L. 464*

Sell, E. J. 470*

Sengers, R. C. A. 598

Senior, B. $374, * 438 *$

Serine 598, 941

Serine hydroxymethylase 721

Seriogina, V. 914 *

Serratto, M. 354*

Sertoli cells 968

Seungdamrong, S. $399 *$

Sevanian, A. $244,362 *$

Sever, J. L. $418^{*}$

Severson, M. V. 452*

Shah, N. 410*

Shah, N. R. $408^{*}$

Shah, U. 417*

Shahin, B. 458*

Shanfield, B. 394*

Shannon, D. C. $470^{*}$

Shanske, A. 395*

Shanske, S. 395*

Shapira, E. 395*

Shapiro, B. 375*

Shapiro, B. H. $369^{*}$

Shapiro, J. R. 400*

Shashikumar, V. 455*

Shaw, J. C. L. 896*

Sheikholislam, B. M. 375*

Shende, A. 396*

Shepherd, J. A. 386*

Sherman, J. O. 386*

Sherwood, W. G. 360,*438*

Shih, V. E. 391

Shin, H. S. 421 *

Shope, T. C. 414*

Shore, S. L. 419*

Shott, R. J. 437,* 441,*448*

Shouraie, Z. 363*

Shukla, S. B. 409*

Shulman, S. T. 418 *

Shumak, K. 412*

Shurin, P. A. 428*

Shwachman, H. $350, * 351, * 355, * 383$, $430 *$

Siassi, B. $444, * 452 *$

Sieber, O. F. $418, * 419 *$

Siefert, C. $375^{*}$

Siégel, N. J. $428, * 461 *$

Sieger, L. $360^{*}$

Sigstrom, L. 899*

Siimes, M. A. 409*

Silken, A. B. $409 *$

Silverman, N. H. 354 *

Simell, O. 904*

Simmons, J. L. 396*

Simmons, M. A. 830

Simon, R. 404*

Simone, J. V. $357, * 409 *$

Simons, S. M. 409*

Sinaiko, A. R. 461 *

Sinaniotis, C. A. $917 *$

Singer, A. D. $419^{*}$

Singley, J. 403*

Sinha, T. 223

Sinks, L. F. $410^{*}$

Sisk, M. A. 429 *

Sisson, T. R. C. $451, * 647$

Sitarz, A. L. 408*

Sivy, M. 540
Sizonenko, P. C. $898, * 918 *$

Skeletal development 248

Skin 257

Skinfold 215

Slaunwhite, W. R., Jr. 375*

Slaven, B. 647

Slichter, S. J. 416*

Sloan, H. R. 387*

Slotkoff, L. M. 456*

Small intestine 735

Smith, A. 915*

Smith, A. L. $435, * 438 *$

Smith, B. T. 848

Smith, C. H. 697

Smith, D. 710

Smith, D. W. $390, * 440 * * 441 *$

Smith, E. W. P. 429*

Smith, F. G., Jr. $460, * 611$

Smith, G. 357*

Smith, J. Z. 430*

Smith, K. A. 409*

Smith, K. O. 415*

Smith, M. R. 421*

Smith, R. E. 353*

Smithwick, E. M. 394 *

Smoking 960

Snead, C. $405^{*}$

Snyder, E. 346*

Snyder, R. 465*

Sobel, E. H. 395*

Sochor, V. $411 *$

Sodium reabsorption 861

Sodt, P. 348*

Soleymani, Y. 465*

Somatomedin 163

Somers, L. 455*

Sommer, A. 442*

Soothill, J. F. 901*

Soriano, H. 359,* $382 *$

Southard, J. L. 389,* $899 *$

Sovik, O. $918^{*}$

Sparkes, R. S. 393*

Spaulding, D. R. 387*

Speck, W. T. 451*

Speckmann, E. W. 837

Spence, L. 427

Spence, M. W. 628

Spencer, F. 78

Sperling, M. 435*

Sperling, M. A. $356, * 432, * 439, * 951$

Spermidine 231

Sphingomyelin 89

Spicer, S. S. 467*

Spitzer, A. $453, * 455, * 458, * 459 *$

Spitzer, R. $419, * 461 *$

Spitzer, R. E. 420*

Spotnitz, H. M. $355^{*}$

Spranger, J. $904{ }^{*} 916^{*}$

Spranger, J. W. $440^{*}$

Sprunt, K. 422,* 429*

Stacy, T. E. $899 *$

Staehr-Johansen, K. 412*

Stagno, S. 428, $430 *$

Stahl, M. K. 689

Stahlman, M. T. 470*

Stalder, G. 903*

Stanecky, O. J. $390 *$

Stanger, P. 352*

Stark, G. 361*

Starling, K. A. $400^{*}$ 
Starr, S. E. $419^{*}$

Starzl, T. E. 379*

Staub, N. C. $464 *$

Steeg, C. N. 467*

Steele, M. W. 396*

Steele, R. W. $413, * 420 * 421 *$

Steendijk, R. 897*

Stegink, L. D. 386*

Steichen, J. J. 451*

Steinfeld, L. 445*

Steinmann, B. $910^{*}$

Ste-Marie, M. 385*

Ste-Marie, M. T. 815

Stenzel, K. H. 163

Stephenson, S. R. 375*

Stern, C. M. M. 918*

Stern, L. $445, * 466^{*}$

Stern, R. C. $422 *$

Stevenson, R. E. $360, * 433 *$

Stewart, J. M. 414*

St. Geme, J. W., Jr. 421*

Stickler, G. B. 453*

Stiehm, E. R. $417, * 420^{*}$

Still, W. J. S. 405*

Stillwell, W. G. $369^{*}$

Stinson, D. A. $443^{*}$

Stites, L. J. $368^{*}$

Stitzel, A. $419, * 461 *$

Stockman, J. A. $409, * 410, * 420, * 439 *$

Stockman, J. A., III 410 *

Stoner, G. E. 75

Storer, J. S. 429*

Strandberg, K. 908*

Strangway, A. 354*

Strauch, B. 428*

Strauss, H. 406*

Strauss, J. $461^{*}$

Strauss, M. E. $365^{*}$

Streissguth, A: P. $440^{*}$

Strife, C. F. 461*

Ströder, J. 897*

Stuart, M. J. $409, * 410, * 420, * 439 *$

Stubbs, K. G. 428*

Sturman, J. A. 357 *

Sulfatides 89

Sulis, C. $440^{*}$

Summitt, R. L. $369, * 442 *$

Summons, R. 446*

Sumners, J. E. 215

Sundaram, S. K. $448^{*}$

Sunshine, P. 5

Surfactant 874

Suter, S. N. $375^{*}$

Sutherland, J. M. 342*

Svenningsen, N. N. 659, $895^{*}$

Sweet, A. Y. $383, * 444, * 448 *$

Swender, P. T. $346 *$

Swerdloff, R. S. 368*

Swierczewski, E. 5

Swyer, P. R. 449, * 469 *

Szczepanik, P. 366*

Szelid, Z. S. $898^{*}$

Szygenda, S. A. $384^{*}$

Szymanski, M. 379*

Tada, K. 72

Taeusch, W., Jr. 467*

Taghizadeh, A. 896*

Tai, Y. H. $898, * 909$

Tai, Y. J. $380^{*}$
Take, H. 403*

Tallan, H. 432*

Tallan, H. H. 388*

Talner, N. S. $349 *$

Tamer, M. A. 429 *

Tan, C. 408*

Tanaka, K. $387, * 440^{*}$

Tang, S. C. $367 *$

Tanner, J. M. 149, 157

Tanprasert, K. 386, ${ }^{*} 459 *$

Taqi, Q. 358*

Tattoni, D. S. $375^{*}$

Taunay, A. d. E. 429*

Taussig, L. M. 468, ${ }^{*} 470^{*}$

Taylor, A. $459, * 861$

Taylor, A. L. 376*

Taylor, F. H. 408*

Taylor, P. M. 443*

Teasdale, F. $360^{*}$

Teberg, A. 451*

Tedesco, F. J. $381 *$

Teitelbaum, H. 343*

Tenenhouse, H. 396*

Tennes, K. 394*

Tenore, A. $370 * 371, * 376 * 377 *$

Terasaki, P. I. 417 *

Testis 755

Tetrault, S. M. $410^{*}$

Thach, B. T. 471*

Thalassemia 553

$\alpha$-Thalassemica 955

Thaler, M. M. 401*

Thau, R. 1

Thau, R. B. $448^{*}$

Thibeault, D. 354*

Thier, S. O. 673

Thilenius, O. G. 67

Thoenes, W. 902*

Thomasson, B. H. 380*

Thompson, J. B. 710

Thong, Y. H. 139, 140

Thornton, N. H. $344 *$

Thymidine Kinase 843

Thyroid 783

Tibbles, J. A. R. 628

Tiglic acid 941

Tina, L. $458^{*}$

Ting, R. 346*

Tinschmann, P. 82

Tischier, V. 914, * 917 *

Tissue culture 746

Tittle, K. L. $400^{*}$

Tiwary, C. M. 376 *

Tocci, P. M. 376* 386 *

Todd, J. L. $355^{*}$

Toews, C. J. 438*

Tolksdorf, C. M. $904, * 916 *$

Tomar, R. H. 420*

Tooley, W. A. 358*

Tooley, W. H. 354*

Torday, J. S. 848

Torrado, A. $894, * 896 *$

Total body potassium 879

Toubas, P. L. 451*

Towell, M. E. 471*

Traggis, D. $402 *$

Trainor, E. J. $364 *$

Transfer RNA 205

Transport 884

Trause, M. A. $340 *$
Travers, R. 381, 389*, 893,*899*

Travis, K. W. 469*

Travis, L. B. 454*

Triglyceride 114

Trijbels, J. M. F. 598

Trinidad, E. E. $438^{*}$

Tronick, E. C. $342 *$

Truccone, N. J. $355^{*}$

Trygstad, C. W. 223, 368*

Tsai, P. 451*

Tsang, H. L. 381*

Tsang, R. 434*

Tsang, R. C. $376, * 451 *$

Tsao, M. U. 433*

Tsiantos, A. 430*

Ts'o, T. O. T. 396*

Tucker, C. R. $350, * 351, * 355 *$

Tunick, F. 348*

Tunnessen, W. W., Jr. 346*

Turgeon, L. 342*

Turner, S. W. 469*

Tuvemo, T. 908*

Typhoid vaccine 809

Tyrosine 714

Tyrosine hydroxylase 714

Tyrosine transaminase 37

Umbilical cord 821

Underwood, L. E. 376*

Unglaub, W. G. 652

Upton, G. V. 435*

Urea 830

Uremia 109, 546

Urine 223

Urmson, J. R. 406, *410*

Ungefehr, K. 900*

Utiger, R. 372*

Utiger, R. D. $371^{*}$

Uy, C. G. 402*

Vaidya, V. $370-372, * 376,377 *$

Vaisman, S. $366^{*}$

Vallbona, C. $345^{*}$

Valle, D. L. 396*

Vallet, H. L. 377*

Vallo, A. 895*

Van Damme, B. 902*

Van Den Berghe, G. $910^{*}$

Vanderschueren-Lodewey ckx, M. 800 van der Zee, S. P. M. 598

Vanmandelic acid 809

Vannucci, R. C. $464^{*}$

Van Wieringen, P. M. V. $896^{*}$

Van Wyk, J. J. 376*

Varga, F. 357,* $911 *$

Varghese, P. J. $347, * 352 *$

Vargo, T. A. $355^{*}$

Varma, A. 342*

Vasopressin 223

Vawter, G. F. 381*

Ventricular volume 67

Vergalla, J. M. 573

Vernier, R. L. 461*

Versmold, H. 907*

Verzosa, M. 409*

Vesikari, T. 420*

Vickers, P. 413*

Vidailhet, M. 393*

Vidnes, J. 918*

Vidyasagar, D. $437, * 443 *$ 
Vila, A. $895,{ }^{*} 913 *$

Villareale, M. E. 439*

Vinas, C. $367^{*}$

Vincent, M. M. 414, 420 *

Virnig, N. L. 452*

Vischer, D. $905^{*}$

Viseskul, C. $439, * 441 *$

Visintine, A. M. 419*

Visy, M. 914*

Vitale, L. F. $410^{*}$

Vitamin $B_{6} 546$

Vladimer, G. M. $430^{*}$

Vlatkovic, G. 915*

Vocalizations 921

Voeller, K. 367*

Vogt, J. F. 452*

Voina, S. J. 376*

Volk, T. L. $375^{*}$

Völlmin, J. A. 704

von Bassewitz, D. B. 82

von Behrens, W. 416*

Voorhess, M. L. 393,* 439*

Vrenken, J. A. Th. 598

Vyden, J. K. 393*

Waggoner, J. G. 573

Wagner, H. 357*

Wai Kan, Y. 553

Wakid, N. W. $909^{*}$

Waldman, J. D. $355^{*}$

Waldman, S. $452 *$

Walker, R. D. $424,{ }^{*} 455,{ }^{*} 460 *$

Walker, W. A. $420^{*}$

Wallach, L. A. 363*

Walling, L. $430^{*}$

Walravens, P. A. 386 *

Walters, L. R. 353*

Wang, J. J. $410^{*}$

Wangensteen, O. D. $468, * 621$

Wannamaker, L. W. 424*

Wanner, A. $471^{*}$

Wapnir, R. A. $380, * 383 *$

Wappner, R. S. 388*

Wara, D. W. $420, * 463 *$

Ward, R. H. T. $899^{*}$

Warner, M. 366*

Warren, B. L. 421 *

Warren, R. $425 *$

Warshaw, J. B. 193, 361,*440, * 445*

Warshawsky, J. T. 471*

Warwick, W. J. 471*

Wasserman, E. 346*

Wasserman, R. H. 439*

Watkins, J. B. $366^{*}$

Watson, J. H. L. 442*

Watts, H. 457*

Weaning 149,157

Weber, A. $385^{*}$
Weber, A. M. $385^{*}$

Wedgwood, R. J. 416*

Wehrle, P. 422*

Weichsel, M. E., Jr. $361,{ }^{*} 843$

Weidman, W. H. $353^{*}$

Weinberg, A. G. $460^{*}$

Weiner, J. $400^{*}$

Weiner, L. B. 430 *

Weiner, L. S. $410^{*}$

Weinfeld, I. J. 452*

Weinstein, A. 419*

Weismann, U. N. 865

Weiss, L. 418* $442 *$

Weiss, N. S. $465^{*}$

Weisz, L. 464*

Weitzman, M. 407*

Welch, J. P. 391 *

Wendel, U. 904, * 906*

Wennberg, R. 963

Wentrup, H. 906*

Werder, E. A. 248

West, C. D. $461^{*}$

West, R. J. 911*

Westcott, S. 908*

Weston, C. $377^{*}$

Wexler, J. R. $346^{*}$

Wharton, B. A. 918*

Whaun, J. M. 411*

Wheeler, M. 349*

Whitaker, T. R. 437*

White, J. G. 421*

Whitelaw, W. 467*

Whitley, R. J. 423*

Whitman, V. 465*

Whitten, D. M. 421*

Wickler, M. 451*

Wiebe, R. A. 421*

Wiedemann, E. 163, 374*

Wiesmann, U. N. 531, 904* 908*

Wiktor, T. $417 *$

Wiley, B. 425*

Wiley, H. E., III 442*

Wiley, J. S. $411^{*}$

Wilfert, C. M. $430^{*}$

Wilkinson, C. $346^{*}$

Williams, J. P. G. 149, 157

Williams, M. L. $393, * 437, * 448 *$

Williams, P. R. 432,*439*

Williams, R. G. $350, * 355, * 430 *$

Willis, D. M. $452 *$

Willis, R. A. 652

Wilroy, R. S., Jr. 442*

Wilson, C. $430^{*}$

Wilson, C. J. $456^{*}$

Wilson, R. 385*

Wilson, S. $447^{*}$

Wilson, W. M. $469^{*}$

Wilson's disease 821

Wingfield, B. S. 371 *
Winkelstein, J. A. 421*

Winkler, L. 357*

Winterborn, M. 454*

Wiser, W. L. $369^{*}$

Wit, A. L. $350^{*}$

Wolfe, R. 361*

Wolff, J. A. 408*

Wolkoff, A. W. 591

Wong, A. S. $387^{*}$

Wong, P. W. K. 396, * 444 *

Wong, W. H. 257

Wonneberger, B. 18

Wood, J. 349*

Wood, R. E. 452, * 471*

Woodhead, A. P. 403*

Wotman, S. 363*

Wright, L. 931

Wright, P. F. 430*

Wu, M. $420 *$

Wu, P. Y. K. 257

Wu, R. $378^{*}$

Wyss, M. 898*

Wyszogrodski, I. 467, * 471 *

X-linked defect 5

$\mathrm{X}$-linked recessive disorder 170

Yaddanapudi, R. 408*

Yaeger, R. G. 652

Yaffe, S. J. $358, * 364, * 365 *$

Yaish, H. M. 409*

Yamauchi, T. 421*

Yang, H. 366*

Yankee, R. A. 404*

Yanover, M. J. 346*

Yasmin, N. 413*

Yavin, E. 263

Yeh, M. 454*

Yeh, T. 354*

Yoshida, T. 361*

Young, P. 345,*398*

Young, R. B. 378*

Younoszai, M. K. 386*

Yuan, L. 453*

Zaby, V. $366^{*}$

Zachmann, M. 248

Zackai, E. 442*

Zahnd, G. 918*

Zak, R. 348*

Zarif, M. 437*

Zarkowsky, H. S. 407*

Zeiger, R. S. $421^{*}$

Zeilstra, K. 359,*393*

Zetterström, R. 852

Zinkham, W. H. 404*

Zuelzer, W. W. 403*

Zurbrugg, R. P. 909* 